\title{
CARRAPATOS EM ANIMAIS SILVESTRES DO BIOMA CERRADO TRIADOS PELO CETAS, IBAMA-GOIÁS
}

\author{
WILD ANIMALS TICKS IN THE CERRADO BIOME SCREENED BY THE \\ CETAS, IBAMA-GOIÁS
}

\author{
Thiago Souza Azeredo Bastos ${ }^{*}$ \\ Darling Melany de Carvalho Madrid ${ }^{1}$ \\ Adriana Marques Faria ${ }^{1}$ \\ Thais Miranda Silva Freitas ${ }^{1}$ \\ Guido Fontgalland Coelho Linhares ${ }^{1}$ \\ 1Escola de Veterinária e Zootecnia da Universidade Federal de Goiás, Goiânia, GO, Brasil. \\ *Autor para correspondência - tsabvet@gmail.com
}

\section{Resumo}

O Bioma Cerrado possui por volta de 320 mil espécies de animais e ocupa aproximadamente $25 \%$ do território brasileiro. Por sua grande diversidade de espécies, excita o tráfico ilegal de animais silvestres. O Instituto Brasileiro do Meio Ambiente e dos Recursos Naturais Renováveis (IBAMA) apreende animais traficados e encaminha-os para o Centro de Triagem de Animais Silvestres (CETAS), onde passam por triagem, são identificados e submetidos a testes diagnósticos e à quarentena, antes de serem encaminhados a uma destinação final. A identificação de ectoparasitas, possíveis vetores de zoonoses, está entre um dos procedimentos preconizados. Foi realizada, durante o ano de 2013, a colheita e identificação de carrapatos encontrados em animais silvestres encaminhados ao CETAS. As respectivas espécies foram identificadas: Amblyomma longirostre em ouriço-caxeiro (Coendou prehensilis); Amblyomma rotundatum em jiboia (Boa constrictor); Amblyomma sculptum e Rhipicephalus microplus em veado-mateiro (Mazama americana); Amblyomma sculptum em lobo-guará (Chrysocyon brachyurus); Amblyomma sculptum e Amblyomma dubitatum em capivara (Hydrochoerus hydrochaeris); Amblyomma nodosum em tamanduá-mirim (Tamandua tetradactyla); Amblyomma sculptum em bugio (Alouatta guariba). Amblyomma foi o principal gênero encontrado. Este é o gênero mais disperso entre os animais silvestres da América. Animais silvestres frutos de apreensão apresentaram baixo número de carrapatos. Isto pode ter acontecido pela forma como são manejados nos criatórios.

Palavras-chave: Amblyomma; Ectoparasito; Goiás.

\begin{abstract}
Cerrado biome has around 320,000 species of animals and it occupies approximately $25 \%$ of the Brazilian territory. This great number of species stimulates the illegal trafficking of wild animals. The Instituto Brasileiro do Meio Ambiente e dos Recursos Naturais Renováveis (IBAMA) is responsible for rescuing trafficked animals and forwards them to the Centro de Triagem de Animais Silvestres (CETAS), where they are screened, identified, and subjected to diagnostic tests and quarantine before being sent to a final destination. Ectoparasites identification is one of the recommended procedures. During 2013, the tick species were identified in wild animals kept in CETAS: Amblyomma longirostre in porcupine (Coendou prehensilis); Amblyomma rotundatum in common boa (Boa constrictor); Amblyomma sculptum and Riphicephalus microplus in Red brocket deer (Mazama americana); Amblyomma sculptum in maned wolf (Chrysocyon brachyurus); Amblyomma sculptum and
\end{abstract}


Amblyomma dubitatum in capybara (Hydrochoerus hydrochaeris); Amblyomma nodosum in collared anteater (Tamandua tetradactyla); Amblyomma sculptum in brown howler (Alouatta guariba). Amblyomma was the main genre found dispersed among wild animals of South America. Captivity animals showed low number of ticks.

Keywords: Amblyomma; Goiás; Tick.

Enviado em: 22 junho 2015

Aceito em: 10 março 2016

\section{Introdução}

No Brasil, o bioma Cerrado é o segundo maior em extensão, superado apenas pela Amazônia. Ele ocupa aproximadamente $24 \%$ do território brasileiro, abrangendo o Distrito Federal, Goiás e outros nove Estados. Também, possui área de transição com outros quatro biomas brasileiros: Amazônia, Caatinga, Pantanal e Mata Atlântica. Esta característica lhe concede uma alta biodiversidade ${ }^{(1)}$.

Estima-se que no Cerrado ocorram 320 mil espécies de animais diferentes, sendo que 0,6\% representam os vertebrados. Isto representa aproximadamente 935 espécies de aves, 298 mamíferos e 268 espécies de répteis do Brasil ${ }^{(1)}$. Esta diversidade de espécies excita o tráfico ilegal de animais silvestres. Atividade que resultou no encaminhamento de mais de três mil espécimes ao Instituto Brasileiro do Meio Ambiente e dos Recursos Naturais Renováveis - IBAMA no ano de 2010. Deste total, a maior parte foi de aves e os demais pertencem aos grupos dos mamíferos e répteis ${ }^{(2)}$.

De acordo com a Instrução Normativa de $\mathrm{n}^{\circ} 179$ de 2008-IBAMA, os animais capturados pelo IBAMA devem ser encaminhados para o Centro de Triagem de Animais Silvestres - CETAS, onde passarão por triagem, serão identificados e submetidos à quarentena antes de serem encaminhados a uma destinação final. Ainda, segundo a mesma instrução normativa, caso a soltura dos animais seja determinada como destinação final, durante o período em que estes animais permanecem no CETAS, devem-se realizar exames e procedimentos para diagnóstico de diversas enfermidades que possam representar risco para a população humana e animal. A coleta e identificação de ectoparasitos é um destes procedimentos preconizados ${ }^{(3)}$.

Dentre os ectoparasitas, os carrapatos se destacam pela sua abundância e possível transmissão de agentes patogênicos ${ }^{(4)}$. De acordo com Sponchiado et al. ${ }^{(5)}$, estão registradas 66 espécies de carrapatos no Brasil, sendo 45 da família Ixodidae e 21 da família Argasidae.

Ixodídeos, comumente encontrados em animais domésticos e silvestres, destacam-se pelo fato de poderem transmitir doenças de importância sanitária como dermatofilose, babesiose, erlichiose, febre maculosa, febre do Nilo ocidental, febre Q e borreliose ${ }^{(6)}$. Contudo, além destas, ainda existem enfermidades parasitárias que pouco se conhece, principalmente em seu ciclo silvestre, mesmo em regiões cuja ocorrência seja endêmica ${ }^{(7)}$.

Portanto, objetivou-se com este estudo identificar as espécies de carrapatos que parasitam animais silvestres do bioma cerrado para melhor entendimento da relação parasito-hospedeiro.

\section{Material e Métodos}

O projeto foi realizado entre março e novembro de 2013 em Goiânia, Goiás, Brasil. Foram feitas duas visitas mensais ao CETAS-GO, local onde os animais capturados e apreendidos pelo IBAMA, no Estado, são enviados para avaliação clínica, triagem e escolha de destinação apropriada.

Todos os animais depois de registrados e triados, foram contidos e inspecionados para se verificar a presença de carrapatos. Para a colheita, todos os animais foram contidos fisicamente. Após inspeção minuciosa de cada animal, todos os carrapatos encontrados foram removidos manualmente ou 
com auxílio de pinça. Em seguida foram armazenados em frascos plásticos contendo álcool 70\% e encaminhados para o laboratório.

A identificação foi realizada no Laboratório de Doenças Parasitárias do Setor de Medicina Veterinária Preventiva, da Escola de Veterinária e Zootecnia - EVZ da Universidade Federal de Goiás - UFG, que conta com instalações e equipamentos apropriados para tal finalidade. Após esta identificação, os espécimes foram encaminhados para o Instituto Butantan, onde após a identificação ser confirmada, foram incluídos à Coleção Acarológica desta instituição.

Para a identificação dos carrapatos, utilizou-se um microscópio estereoscópico e a chave de classificação de Onofrio et al. ${ }^{(8)}$. A identificação dos animais parasitados foi feita pelos técnicos do CETAS-GO, de forma que os dados apresentados neste trabalho são provenientes das fichas de triagem utilizada pelo estabelecimento.

Todas as coletas foram feitas com autorização do IBAMA, de acordo com o protocolo de número $38315-1 / 2013$.

\section{Resultados}

Durante os meses de março e novembro de 2013, foram inspecionados 132 animais. A maior parte foi recebida pelo CETAS-GO durante os meses de março, abril e maio, totalizando 81 animais (61\%) neste período. Em junho, julho e agosto foram recebidos outros 31 animais (24\%) e, entre setembro e novembro, apenas 20 animais $(15 \%)$ foram inspecionados.

Deste total de animais inspecionados, 44 (33\%) eram mamíferos, $82(62 \%)$ eram aves e seis (5\%) eram répteis. Apenas em oito animais $(6 \%)$ foram encontrados com carrapatos: seis mamíferos, uma ave e um réptil. Destes animais, reuniu-se um total de 26 carrapatos. Dentre eles, 12 espécimes foram colhidos na fase adulta e outros 14 na fase de ninfa. Todos os carrapatos do gênero Amblyomma foram incluídos à Coleção Acarológica do Instituto Butantan, onde receberam as seguintes identificações de tombamento: IBSP 12.467; IBSP 12.468; IBSP 12.469; IBSP 12.470; IBSP 12.471; IBSP 12.472 e IBSP 12.473.

Observou-se que entre março e maio (outono), período de maior entrada de animais no CETAS-GO, foram detectados dois animais parasitados. Todos os outros seis animais parasitados $(75 \%)$ foram recebidos no estabelecimento durante os meses de junho, julho e agosto (inverno). Em setembro, outubro e novembro (primavera), não foram encontrados animais com os ectoparasitos em questão.

Cinco espécies de Amblyomma e uma de Riphicephalus foram identificadas. Os ixodídeos identificados, sua contagem e as respectivas espécies parasitadas foram: Amblyomma longirostre $(\mathrm{n}=3)$ em ouriçocaxeiro(Coendouprehensilis); Amblyomma rotundatum $(\mathrm{n}=1)$ emjiboia(Boa constrictor); Amblyomma sculptum ( $\mathrm{n}=1)$ e $R$. microplus $(\mathrm{n}=3)$ em veado-mateiro (Mazama americana); Amblyomma sculptum $(\mathrm{n}=4)$ em lobo-guará (Chrysocyon brachyurus); Amblyomma sculptum $(\mathrm{n}=7)$ e Amblyomma dubitatum $(\mathrm{n}=3)$ em capivara (Hydrochoerus hydrochaeris); Amblyomma nodosum $(\mathrm{n}=2)$ em tamanduá-mirim (Tamandua tetradactyla); Amblyomma sculptum $(\mathrm{n}=1)$ em bugio (Alouatta guariba). Uma espécie de Amblyomma provenientes de seriema (Cariama cristata) não apresentou condições que permitissem sua identificação (Tabela 1).

Capivara foi a espécie com maior quantidade de carrapatos coletados por indivíduo e $A$. sculptum foi o carrapato colhido em maior quantidade no estudo. Por outro lado, apesar de inspecionados, não foram encontrados carrapatos nos seguintes mamíferos: raposa do campo (Pseudalopex vetulus) $(\mathrm{n}=2)$, jaguatirica (Felis pardalis) $(\mathrm{n}=1)$, sagui-do-tufo-preto (Callithrix penicillata) $(\mathrm{n}=6)$, tatu-folha (Dasypus novemcinctus) $(\mathrm{n}=1)$, irara (Eira barbara) $(\mathrm{n}=1)$, cachorro-do-mato (Cerdocyon thous) $(\mathrm{n}=2)$, quati (Nasua nasua) $(\mathrm{n}=3)$, tamanduá-bandeira (Myrmecophaga tridactyla) $(\mathrm{n}=1)$, gambá (Didelphis albiventris) ( $\mathrm{n}=4)$, gato-do-mato (Leopardus tigrinus)( $\mathrm{n}=1)$ e macaco-prego (Cebus libidinosus) $(\mathrm{n}=1)$. 
Tabela 1: Espécies de carrapatos identificados e seus respectivos hospedeiros.

\begin{tabular}{lllll}
\hline Grupo & Animal parasitado & PI* $^{*}$ & Carrapatos identificados & $\mathbf{Q}^{* *}$ \\
\hline Mamiferos & Coendou prehensilis & $1 / 1$ & Amblyomma longirostre & $2 \mathrm{M}, 1 \mathrm{~F}$ \\
& Mazama americana & $1 / 5$ & Amblyomma sculptum & $1 \mathrm{~F}$ \\
& & $1 / 5$ & Riphicephalus microplus & $1 \mathrm{M}, 2 \mathrm{~F}$ \\
& Chrysocyon brachyurus & $1 / 3$ & Amblyomma sculptum & $4 \mathrm{M}$ \\
& Hydrochoerus hydrochaeris & $1 / 3$ & Amblyomma sculptum & $7 \mathrm{~N}$ \\
& & & Amblyomma dubitatum & $2 \mathrm{M}, 1 \mathrm{~F}$ \\
& Tamandua tetradactyla & $1 / 1$ & Amblyomma nodosum & $2 \mathrm{~F}$ \\
Réptil & Alouatta sp. & $1 / 6$ & Amblyomma sculptum & $1 \mathrm{~N}$ \\
Ave & Boa constrictor & $1 / 1$ & Amblyomma rotundatum & $1 \mathrm{~F}$ \\
& Cariama cristata & $1 / 1$ & Amblyomma sp. & $1 \mathrm{~N}$
\end{tabular}

*Prevalência de infestação $(\mathrm{PI})=$ número de animais parasitados $/$ número de animais inspecionados. ${ }^{* *} \mathrm{Q}=$ quantidade de carrapatos colhidos no animal. $\mathrm{M}=$ macho, $\mathrm{F}=$ Fêmea, $\mathrm{N}=\mathrm{Ninfa}$.

Dentre os répteis, jabuti (Chelonoidis denticulata) $(\mathrm{n}=4)$ e iguana (Iguana iguana) $(\mathrm{n}=1)$ também não apresentaram tais ectoparasitos. Por ultimo, uma diversidade de espécies de aves foi inspecionada, mas nenhum representante das ordens psittaciformes $(n=35)$, passeriformes $(n=32)$, piciformes $(n=8)$, columbiformes $(n=4)$, gruiformes $(n=2)$ e strigiformes $(n=1)$ apresentaram parasitismo por carrapatos.

\section{Discussão}

O estudo foi realizado durante dez meses, entre o final e início do período de chuva, envolvendo todo o período de seca na região centro-oeste do País. Foi observada maior colheita de carrapatos durante o inverno, época de menor umidade relativa do ar na região em questão. Esta informação coincide com outros estudos conduzidos envolvendo animais domésticos e silvestres no mesmo bioma ${ }^{(9)}$.

As aves representaram a maioria dos animais inspecionados, sendo passeriformes e psitaciformes as ordens de maior predominância. Entretanto, em nenhuma ave foram encontrados carrapatos. Labruna et al. ${ }^{(10)}$ mencionaram que pode ser difícil encontrar formas adultas em passeriformes. Ainda assim, é possível que as aves inspecionadas neste estudo, por serem provenientes de cativeiro, tenham recebido algum tipo de tratamento acaricida. $\mathrm{O}$ que justificaria a ausência em quase todos os animais deste grupo.

Segundo Luz et al. $\left({ }^{9}\right)$, em passeriformes no Cerrado, pode-se encontrar grande quantidade de ninfas de $A$. longirostre, A. nodosom e Amblyomma ovale. Labruna et al. ${ }^{(10)}$ também relataram que ninfas de A. longirostre parasita aves, mas adultos são encontrados principalmente parasitando roedores. Isto foi observado no CETAS-GO, onde duas fêmeas e um macho de $A$. longirostre foram detectados em um ouriço-caxeiro (C. prehensilis).

Ainda, conforme Luz et al. ${ }^{(9)}$, A. nodosum foi a terceira espécie mais encontrada na forma de ninfa parasitando aves do Cerrado. Segundo Teixeira et al. ${ }^{(11)}$, sua forma adulta geralmente é encontrada em Xenarthra (Edentata), relação também observada por Bechara et al. ${ }^{(12)}$. Tais informações coincidem com os achados do atual estudo, em que duas fêmeas de $A$. nodosum foram encontradas em um $T$. tetradactyla.

Durante a realização de um trabalho de captura de pequenos mamíferos do Cerrado, Sponchiado et al. ${ }^{(5)}$ também fizeram a coleta de carrapatos. Neste caso, D. albiventris foi a segunda espécie mais capturada, mas foi a que apresentou maior parasitismo, predominando as ninfas de $A$. sculptum e A. coelebs respectivamente. Tais resultados contrastam com os obtidos neste estudo, pois foram 
inspecionados quatro gambás (D. albiventris) no CETAS-GO e nenhum deles apresentou carrapatos. A. sculptum é conhecidamente a espécie de maior ocorrência em capivaras, podendo ser encontrada em uma grande diversidade de outros animais. Neste trabalho, A. sculptum foi recolhido em um exemplar de capivara (H. hydrochaeris), lobo-guará (C. brachyurus), bugio (A. guariba) e veado-mateiro ( $M$. americana). Trabalhos anteriores realizados no estado de Goiás já relataram a presença de $A$. sculptum parasitando C. brachyurus $^{(13)}$. Ainda, é importante ressaltar que $A$. cajennense sensu strictu, espécie morfologicamente muito parecida com A. sculptum, mesmo não detectada neste estudo, também está presente no estado de Goiás ${ }^{(14)}$. Isto demanda maiores cuidados durante a identificação das espécies. $\mathrm{Na}$ única capivara parasitada, a espécie $A$. dubitatum foi encontrada em parasitismo concomitante com A. sculptum. Apesar de existirem relatos em outros mamíferos, incluindo o ser humano ${ }^{(15)}, A$. dubitatum é considerado um parasita específico de capivara ${ }^{(16)}$, podendo ser encontrado em áreas do centro-oeste, sudeste e sul do Brasil.

Um exemplar de $A$. rotundatum foi obtido em uma jiboia (B. constrictor). Esta relação já havia sido observada por Knight ${ }^{(17)}$ durante estudo realizado em região de Cerrado. No estado de Goiás, esta espécie já foi relatada em cascavel, jabuti e sapos ${ }^{(18)}$. O sapo cururu (Rhinella marina e Rhinella schneideri) é a espécie mais observada dentre as comumente identificadas como hospedeiro natural de A. rotundatum ${ }^{(19)}$. Amblyomma dissimile é outra espécie apontada como parasita de sapos, serpentes e lagartos $^{(20)}$. Ainda, segundo Fiorini et al. ${ }^{(21)}$, A. dissimile é a espécie de maior ocorrência em jiboia ( $B$. constrictor). Contudo, isso não foi observado no CETAS-GO.

Três exemplares de $R$. microplus foram encontrados em um veado-mateiro ( $M$. americana). Esta espécie de ectoparasito frequentemente está relacionada a bovinos, mas há relatos de $R$. microplus em várias outras espécies, como a onça pintada (Panthera onca), o cão e o ser humano ${ }^{(22)}$.

Por fim, o pouco quantitativo de carrapatos obtidos nos animais durante a realização deste estudo serve de alerta para estudos posteriores que pretendam utilizar animais provenientes de apreensões. Ainda, durante o estudo não foram detectadas ninfas parasitando as aves. Situação inesperada, que pode ter acontecido devido à dificuldade de visualização das mesmas, principalmente em animais de pequeno porte, ou pelo fato de serem animais apreendidos que anteriormente eram criados em domicílios como pets.

\section{Conclusões}

Este trabalho forneceu novas informações sobre as espécies de carrapatos que podem ser encontradas nos animais silvestres do bioma cerrado. Amblyomma foi o principal gênero encontrado. A constatação de espécies deste gênero em répteis, aves e mamíferos reforça o quanto estes parasitos estão dispersos entre os animais silvestres da América.

Comportamento semelhante pode ser dito com relação à espécie $R$. microplus que, apesar de ter sido encontrada apenas em um veado-mateiro, esta espécie esta amplamente distribuída entre os animais domésticos, podendo também ser detectada em alguns animais silvestres.

Amblyomma sculptum foi a espécie encontrada em maior quantidade, sendo observado parasitando na capivara, no lobo-guará, no bugio e no veado-mateiro.

Animais silvestres frutos de apreensão apresentam baixo número de carrapatos. Isto pode acontecer pela forma como são manejados nos criatórios, com possível controle de ectoparasitos e isolamento.

\section{Referências}

1. BRASIL. Plano de Ação para Prevenção e Controle do Desmatamento e das Queimadas no Cerrado. PP Cerrado, Brasilia. 2010. 112p.

2. Prado LA, Malheiros R. A Perda da biodiversidade do cerrado goiano mediante o tráfico ilegal de fauna 
silvestre. III Congresso Brasileiro de Gestão Ambiental,Goiânia/GO - 19 a 22/11/2012. 2012:12p.

3. BRASIL. Instituto Brasileiro do Meio Ambiente e dos Recursos Naturais Renováveis. Instrução Normativa no 179 de 25 de junho de 2008. Brasília. 2008.

4. Massard CL, Fonseca AH. Carrapatos e doenças transmitidas, comuns ao homem e aos animais. A Hora Veterinária. 2004;135(1):15-23.

5.Sponchiado J, Melo GL, Martins TF, Krawczak FS, Labruna MB, Cáceres NC. Association patterns of ticks (Acari: Ixodida: Ixodidae, Argasidae) of small mammals in Cerrado fragments, western Brazil. Experimental and Applied Acarology. 2015;65(3):389-401.

6. Brites-Neto J, Duarte KMR, Martins TF. Tick-borne infections in human and animal population worldwide. Veterinary World. 2015;8(3):301-15.

7. Costa APd, Costa FB, Labruna MB, Silveira I, Moraes-Filho J, Soares JF, et al. A serological and molecular survey of Babesia vogeli, Ehrlichia canis and Rickettsia spp. among dogs in the state of Maranhão, northeastern Brazil. Revista Brasileira de Parasitologia Veterinária. 2015;24(1):28-35.

8. Onofrio VC, Labruna MB, Pinter A, Giacomin FG, Barros-Battesti DM. Comentários e chaves para as espécies do gênero Amblyomma in. Barros-Battesti D, Arzua M, Bechara G Carrapatos de importancia medico veterinaria da regiao neotropical Um guía ilustrado para identificaçao de espécies Sao Paulo: Butantan. 2006:53-113.

9. Luz HR, Faccini JLH, Landulfo GA, Berto BP, Ferreira I. Bird ticks in an area of the Cerrado of Minas Gerais State, southeast Brazil. Experimental and applied acarology. 2012;58(1):89-99.

10. Labruna MB, Sanfilippo LF, Demetrio C, Menezes AC, Pinter A, Guglielmone AA, et al. Ticks collected on birds in the state of São Paulo, Brazil. Experimental and Applied Acarology. 2007;43(2):147-60.

11. Teixeira RHF, Miranda FR, Kluyber D, Lopes AMC, Ferreira VL, Guedes NMR, et al. IXODID FAUNA IN EDENTATA (MAMMALIA: XENARTHRA) IN BRAZIL. Revista Saúde Física \& Mental-ISSN 23171790. 2014;3(2):59-73.

12. Bechara GH, Szabó MPJ, Almeida Filho WV, Bechara JN, Pereira RJG, Garcia JE, et al. Ticks associated with armadillo (Euphractus sexcinctus) and anteater (Myrmecophaga tridactyla) of Emas National Park, State of Goias, Brazil. Annals of the New York Academy of Sciences. 2002;969(1):290-3.

13. Labruna MB, Jorge RSP, Sana DA, Jácomo ATA, Kashivakura CK, Furtado MM, et al. Ticks (Acari: Ixodida) on wild carnivores in Brazil. Experimental \& applied acarology. 2005;36(1-2):149-63.

14. Martins TB, ARM; Costa, FB; Peterka, CRL; Pacheco, RC; Terassini, F; Camargo, LMA; Dias, RA; Scofield, A; Campos, AK; Ramirez, DG; Barros-Battesti, DM; Labruna, MB. Estudo do complexo Amblyomma cajennense ( Acari : Ixodidae ) no Brasil RESUMOS APRESENTAÇÃO ORAL - BIOLOGIA BEPA, Bol epidemiol paul (Online) 2013;10(117):8-13.

15. Labruna MB, Pacheco RC, Ataliba AC, Szabó MPJ. Human parasitism by the capybara tick, Amblyomma dubitatum (Acari: Ixodidae). Entomological news. 2007;118(1):77-80.

16. Mastropaolo M, Aprile G, Solis G, Beldomenico PM, Peralta JL, Guglielmone AA. Primer registro de Amblyomma dubitatum Neumann 1899 (Acari: Ixodidae) en la provincia de Misiones (Argentina). Revista FAVE. 2004;3:67-70.

17. Knight JC. Observations on potential tick vectors of human disease in the Cerrado region of central Brazil. Revista da Sociedade Brasileira de Medicina Tropical. 1992;25(2):145-6.

18. Olegário MMM, Szabó MPJ, Santos ALQ. Carrapatos em áreas do cerrado brasileiro. Veterinária Notícias. 2006;12(2):39.

19. Guglielmone AA, Nava S. Hosts of Amblyomma dissimile Koch, 1844 and Amblyomma rotundatum 
Koch, 1844 (Acari: Ixodidae). Zootaxa. 2010;2541:27-49.

20.Lampo M, Rangel Y, Mata A. Population genetic structure of a three-host tick, Amblyomma dissimile, in eastern Venezuela. The Journal of parasitology. 1998:1137-42.

21. Fiorini LC, Craveiro AB, Mendes MC, Chiesorin Neto L, Silveira RD. Morphological and molecular identification of ticks infesting Boa constrictor (Squamata, Boidae) in Manaus (Central Brazilian Amazon). Revista Brasileira de Parasitologia Veterinária. 2014;23(4):539-42.

22. Labruna MB, de Paula CD, Lima TF, Sana DA. Ticks (Acari: Ixodidae) on wild animals from the PortoPrimavera hydroelectric power station area, Brazil. Memórias do Instituto Oswaldo Cruz. 2002;97(8):1133-6. 